\title{
Universality of non-equilibrium fluctuations in strongly correlated quantum liquids
}

\author{
Meydi Ferrier ${ }^{1,2 \star}$, Tomonori Arakawa1, Tokuro Hata ${ }^{1}$, Ryo Fujiwara ${ }^{1}$, Raphaëlle Delagrange ${ }^{2}$, \\ Raphaël Weil $^{2}$, Richard Deblock ${ }^{2}$, Rui Sakano ${ }^{3}$, Akira Oguri ${ }^{4}$ and Kensuke Kobayashi ${ }^{1 \star}$
}

Interacting quantum many-body systems constitute a fascinating research field because they form quantum liquids with remarkable properties and universal behaviour'. In fermionic systems, such quantum liquids are realized in helium-3 liquid, heavy fermion systems ${ }^{1}$, neutron stars and cold gases $^{2}$. Their properties in the linear-response regime have been successfully described by the theory of Fermi liquids ${ }^{1}$. The idea is that they behave as an ensemble of non-interacting 'quasi-particles'. However, non-equilibrium properties have still to be established and remain a key issue of many-body physics. Here, we show a precise experimental demonstration of Landau Fermi liquid theory extended to the non-equilibrium regime in a zero-dimensional system. Combining transport and ultra-sensitive current noise measurements, we have unambiguously identified the SU(2) (ref. 3) and SU(4) (refs 4-8) symmetries of a quantum liquid in a carbon nanotube tuned in the universal Kondo regime. Whereas the free quasi-particle picture is found valid around equilibrium ${ }^{9}$, an enhancement of the current fluctuations is detected out of equilibrium and perfectly explained by an effective charge induced by the residual interaction between quasi-particles ${ }^{8,10-17}$. Moreover, an as-yet-unknown scaling law for the effective charge is discovered, suggesting a new non-equilibrium universality. Our method paves a new way to explore the exotic nature of quantum liquids out of equilibrium through their fluctuations in a wide variety of physical systems ${ }^{18}$.

The Kondo effect ${ }^{19}$ is a typical example of a quantum manybody effect, where a localized spin is screened by the surrounding conduction electrons at low temperature to form a unique correlated ground state. The Kondo state is described well by the Fermi liquid theory at equilibrium ${ }^{1,9}$, which makes it an ideal testbed to go beyond equilibrium. To unveil the universal behaviour of nonequilibrium Fermi liquid ${ }^{11}$, we have used the current fluctuations or shot noise in a Kondo-correlated nanotube quantum $\operatorname{dot}^{18}$.

When electrons are transmitted through this system, the scattering induces the shot noise, which sensitively reflects the nature of the quasi-particles ${ }^{20}$, as shown in the upper panel of Fig. 1a. A remarkable prediction of the non-equilibrium Fermi liquid theory is that the residual interaction between quasi-particles creates an additional scattering of two quasi-particles which enhances the noise (see the lower panel of Fig. 1a) ${ }^{10,12-15}$. This two-particle scattering is characterized by an effective charge $e^{*}$ larger than $e$ (electron charge). This value, closely related to the Wilson ratio, is universal for the Fermi liquid in the Kondo regime as it depends only on the symmetry group of the system ${ }^{13-15}$. Although some aspects of Kondo-associated noise have been reported ${ }^{8,16,17}$, a rigorous, selfconsistent treatment in a regime where universal results apply is at the core of the present work. Actually, by investigating the same nanotube quantum dot in the spin degenerate SU(2) Kondo regime and in the spin-orbit degenerate $\mathrm{SU}(4)$ regime, the noise is proved to contain distinct signatures of these two symmetries, confirming theoretical developments of Fermi liquid theory out of equilibrium.

In our experiment, we measured the conductance and current noise through a carbon nanotube quantum dot grown by chemical vapour deposition ${ }^{21}$. Iron catalyst was deposited on an oxidized undoped silicon wafer and exposed to 10 mbar of acetylene for $9 \mathrm{~s}$ at $900{ }^{\circ} \mathrm{C}$. The nanotube was connected with a $\mathrm{Pd}(6 \mathrm{~nm}) / \mathrm{Al}(70 \mathrm{~nm})$ bilayer deposited by e-gun evaporation. The distance between the contacts is $400 \mathrm{~nm}$ and a side gate electrode is deposited to tune the potential of the quantum dot (see Fig. 1b). A magnetic field of $0.08 \mathrm{~T}$ is applied to suppress superconductivity of the contacts. To measure accurately the shot noise, our sample is connected to a resonant $(2.58 \mathrm{MHz}) \mathrm{LC}$ circuit thermalized at the mixing chamber of the dilution fridge. The signal across this resonator is amplified with an in-house-built cryogenic low-noise amplifier ${ }^{22}$ thermalized on the $1 \mathrm{~K}$ pot and again at room temperature. The power spectral density of the noise is obtained by fast Fourier transformation of the time-domain signal acquired by a digitizer (National Instruments PCI-5922). The current noise of the sample is extracted from the fit of the shape of the resonance in the frequency domain.

Figure 2a presents the image plot of the differential conductance of the sample $(G)$ at temperature $T=16 \mathrm{mK}$ as a function of sourcedrain voltage $\left(V_{\text {sd }}\right)$ and gate voltage $\left(V_{\mathrm{g}}\right)$. This stability diagram shows the fourfold-degenerated Coulomb diamonds specific to carbon nanotubes. The spectrum consists of successive fourelectron shells. We denote by $N=0,1,2,3$ the number of electrons in the last shell. Remarkably, the SU(2) and SU(4) Kondo ridges ${ }^{6}$ emerge as horizontal bright regions (high conductance) at $V_{\mathrm{sd}}=0$.

For the moment, we concentrate on the SU(2) region. A cut of the conductance at $V_{\text {sd }}=0$ is represented in the upper panel of Fig. $2 \mathrm{~b}$. Two Kondo ridges appear as plateaux where $G$ is maximum for the fillings $N=1$ (ridge A) and $N=3$ (ridge B), whereas $G$ decreases to almost zero for even $N$. In addition, the ridge $\mathrm{B}$ is flat and the unitary limit is achieved: the conductance reaches the quantum of conductance $G_{\mathrm{Q}}=2 e^{2} / h$, which is a signature of the perfect Kondo effect in a dot with symmetric coupling to the leads. The Kondo temperature $\left(T_{\mathrm{K}}\right)$ is $1.6 \pm 0.05 \mathrm{~K}$ in the centre of this ridge (see Supplementary Information).

${ }^{1}$ Department of Physics, Graduate School of Science, Osaka University, 1-1 Machikaneyama, Toyonaka, 560-0043 Osaka, Japan. ${ }^{2}$ Laboratoire de Physique des Solides, CNRS, Univ. Paris-Sud, Université Paris Saclay, 91405 Orsay Cedex, France. ${ }^{3}$ The Institute for Solid State Physics, The University of Tokyo,

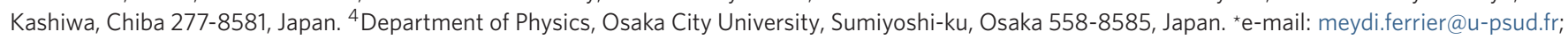
kensuke@phys.sci.osaka-u.ac.jp 
a
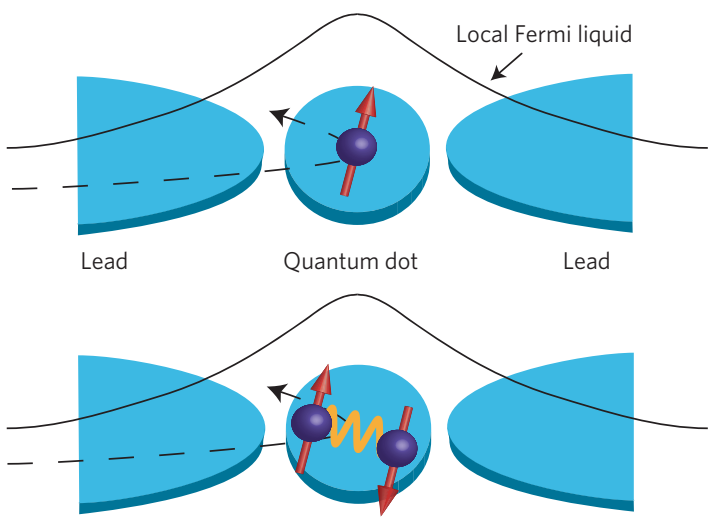

b

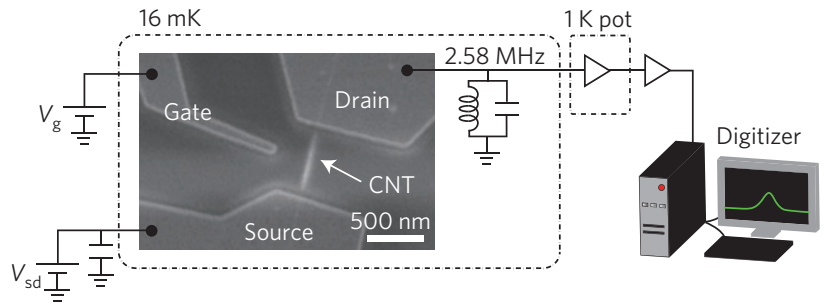

Figure 1 | Illustration of the interaction effect in the quantum liquid and design of the sample. $\mathbf{a}$, Scheme of a quantum dot coupled to two leads. If a single spin is confined on the dot and the coupling with the leads is strong enough, the Kondo effect occurs. The full black line represents the extension of the Kondo state where electrons form a strongly correlated Fermi liquid. Our work consists in a collision experiment. We inject electrons through this state and observe through the current fluctuations which quasi-particles are backscattered. (Top) At low energy, this liquid can be understood as an ensemble of non-interacting quasi-particles. During a collision, only single quasi-particles are excited and backscattered by an incoming electron. (Bottom) At higher energy the incoming electron can excite two quasi-particles of the Fermi liquid through the residual interaction between quasi-particles (orange wavy line). The scattered current consists then of single or double quasi-particle shots which increase the current fluctuations. The effective charge $e^{*}$ is a measure of the probability for these two processes. The Wilson ratio $R$ quantifies the strength of the interaction between two quasi-particles. $\mathbf{b}$, Sample and experimental set-up. SEM image of a carbon nanotube connected to metallic leads on a silicon wafer. It is connected to the current and noise measurement set-up through a resonant circuit fixed on the mixing chamber of the dilution fridge. The gate voltage $V_{g}$ tunes the number of electrons $(N)$ in the dot and the coupling between dot and leads.

The current noise $S_{i}$ and $G$ are plotted in Fig. 2c,d for $N=2$ and $N=3$, respectively, as a function of the source-drain current $I_{\text {sd }}$. Outside the Kondo ridge $(N=2) S_{i}$ is linear with $\left|I_{\mathrm{sd}}\right|$, whereas on the ridge the shot noise is flat around $I_{\mathrm{sd}}=0$ and enhanced at high current when the energy of incoming electrons approaches a fraction of $T_{\mathrm{K}}$. At high voltage $\left(e V_{\mathrm{sd}} \gg k_{\mathrm{B}} T_{\mathrm{K}}\right)$, a linear behaviour is recovered, with $S_{i}=2 e\left|I_{\text {sd }}\right|$. To analyse the low-energy properties, we have extracted the Fano factor $(F)$, which is defined as $S_{i}=2 e F\left|I_{\text {sd }}\right|$, from a linear fit at low current. $F$ varies from approximately zero on the Kondo ridge to $F=1$ outside, as shown in the bottom panel of Fig. 2b. Indeed, at very low energy, as the free quasi-particle picture of the Fermi liquid theory teaches us, the conductance and the noise for a multichannel conductor can be written as a function of transmission $T_{i}$ for each channel $i=1,2,3 \ldots$ (ref. 20):

$$
G=G_{\mathrm{Q}} \sum T_{i} \quad \text { and } \quad F=\frac{G_{\mathrm{Q}}}{G} \sum T_{i}\left(1-T_{i}\right)
$$

For the $\mathrm{SU}(2)$ symmetry, transport occurs through one single channel, yielding $G=G_{\mathrm{Q}} T_{1}$ and $F=1-T_{1}$. On the Kondo ridge, the conductance is $G=G_{\mathrm{Q}}$, yielding $T_{1}=1$ and $F=1-T_{1}=0$. This is a direct signature of the Kondo resonance which allows a perfect transmission and thus no partition of quasi-particles near equilibrium. In the Coulomb blockade regime, for even $N$, the transport is blocked $\left(T_{1} \ll 1\right)$, yielding $F \approx 1$, indicating that transport occurs through tunnelling events resulting in conventional Poissonian noise.

At higher voltages $\left(0<e V_{\text {sd }} \leq k_{\mathrm{B}} T_{\mathrm{K}} / 2\right)$ we concentrate only on the nonlinear terms for current and noise by subtracting the linear part. We defined $S_{\mathrm{K}}=S_{i}-2 e F\left|I_{\mathrm{sd}}\right|$ and the backscattered current $I_{\mathrm{K}}=G(0) V_{\mathrm{sd}}-I_{\mathrm{sd}}$ (refs 10,13). These quantities are related through the effective charge $e^{10,12,13,23} e^{*}$ :

$$
S_{\mathrm{K}}=2 e^{*}\left|I_{\mathrm{K}}\right|
$$

This effective charge does not imply an exotic charge, as in the quantum Hall regime, but is related to the probability that one particle or two particles are backscattered in the Fermi liquid (see Supplementary). Figure 3a,b shows the evolution of the conductance on ridge $\mathrm{B}$ as a function of magnetic field $B$ and the corresponding noise $S_{\mathrm{K}}$ as a function of $I_{\mathrm{K}}$, respectively. The temperature dependence is analysed in detail in the Supplementary Information. The effective charge is directly given by the slope at low current $I_{\mathrm{K}}\left(e V_{\mathrm{sd}}<k_{\mathrm{B}} T_{\mathrm{K}}\right)$, yielding for the lowest field and temperature $e^{*} / e=1.7 \pm 0.1$. This result is in good agreement with theory ${ }^{10,12}$, which predicts $e^{*} / e=5 / 3 \approx 1.67$, corresponding to equal probabilities for one- or two-particle scattering. Figure $3 \mathrm{c}$ represents the evolution of $e^{*}$ with magnetic field and temperature. On this graph $e^{*}$ is represented as a function of the reduced scales $T / T_{\mathrm{K}}$ for temperature or $g \mu_{\mathrm{B}} B / 2 k_{\mathrm{B}} T_{\mathrm{K}}$ for the field, where $g=2$ is the Landé factor and $\mu_{\mathrm{B}}$ the Bohr magneton (see Supplementary Information). All the data points seem to fall on the same curve, suggesting that $e^{*}$ obeys a logarithmic scaling law which has not yet been predicted.

The Wilson ratio $R$ has been extracted from the formula ${ }^{12,15}$

$$
\frac{e^{*}}{e}=\frac{1+9(n-1)(R-1)^{2}}{1+5(n-1)(R-1)^{2}}
$$

where $n$ characterizes the symmetry group $\mathrm{SU}(n)$ of the Kondo state. This number $R$ is directly related to the ratio $U / \Gamma$, with $U$ the charging energy of the quantum dot and $\Gamma$ the coupling to the electrodes. It reflects the strength of the interaction in the Fermi liquid and is the only parameter to characterize the system, going from $R=1$ in the non-interacting case $(U=0)$ to $R=2$ in the strong $\mathrm{SU}(2)$ Kondo limit $(U \rightarrow \infty)$ (see Fig. 3d). Our value for $e^{*}$ yields $R=1.95 \pm 0.1$, ensuring strong interactions and thus a universal regime.

For consistency, $R$ was independently extracted by fitting the evolution of conductance with $B, V_{\text {sd }}$ and $T$ at low excitation (see Supplementary Information) without any assumption on $T_{\mathrm{K}}$. This result, $R=1.95 \pm 0.1$, agrees perfectly with the value extracted from $e^{*}$. Finally, from the dependence of $T_{\mathrm{K}}$ with gate voltage, we have independently extracted the values $U=6 \pm 0.5 \mathrm{meV}$ and $\Gamma=1.8 \pm 0.2 \mathrm{meV}$. This consistency is illustrated in Fig. $3 \mathrm{~d}$, where the two independent values for $R$ and $U / \Gamma$ cross on the theoretical curve.

The effect of asymmetric lead-dot coupling was tested on the ridge A, where $G=0.85 G_{\mathrm{Q}}$. Asymmetry is defined by the factor $\delta$ such that $G(0)=(1-\delta) G_{\mathrm{Q}}$. We have measured an effective charge $e^{*} / e=1.2 \pm 0.08$, in good agreement with ref. 14 , which predicts $e^{*} / e=5 / 3-(8 / 3) \delta=1.26$.

Now, what can we learn from the SU(4) symmetry emerging on the right-hand side of Fig. 2a? A zoom is plotted in the upper panel of Fig. $4 \mathrm{a}$ and a cross-section is shown in the middle panel. Because spin and orbital degrees of freedom are degenerated, two channels contribute to transport, and Kondo resonance emerges at every filling factor, $N=1,2$ and 3 electrons ${ }^{4-7}$. At odd filling, the channels 
a
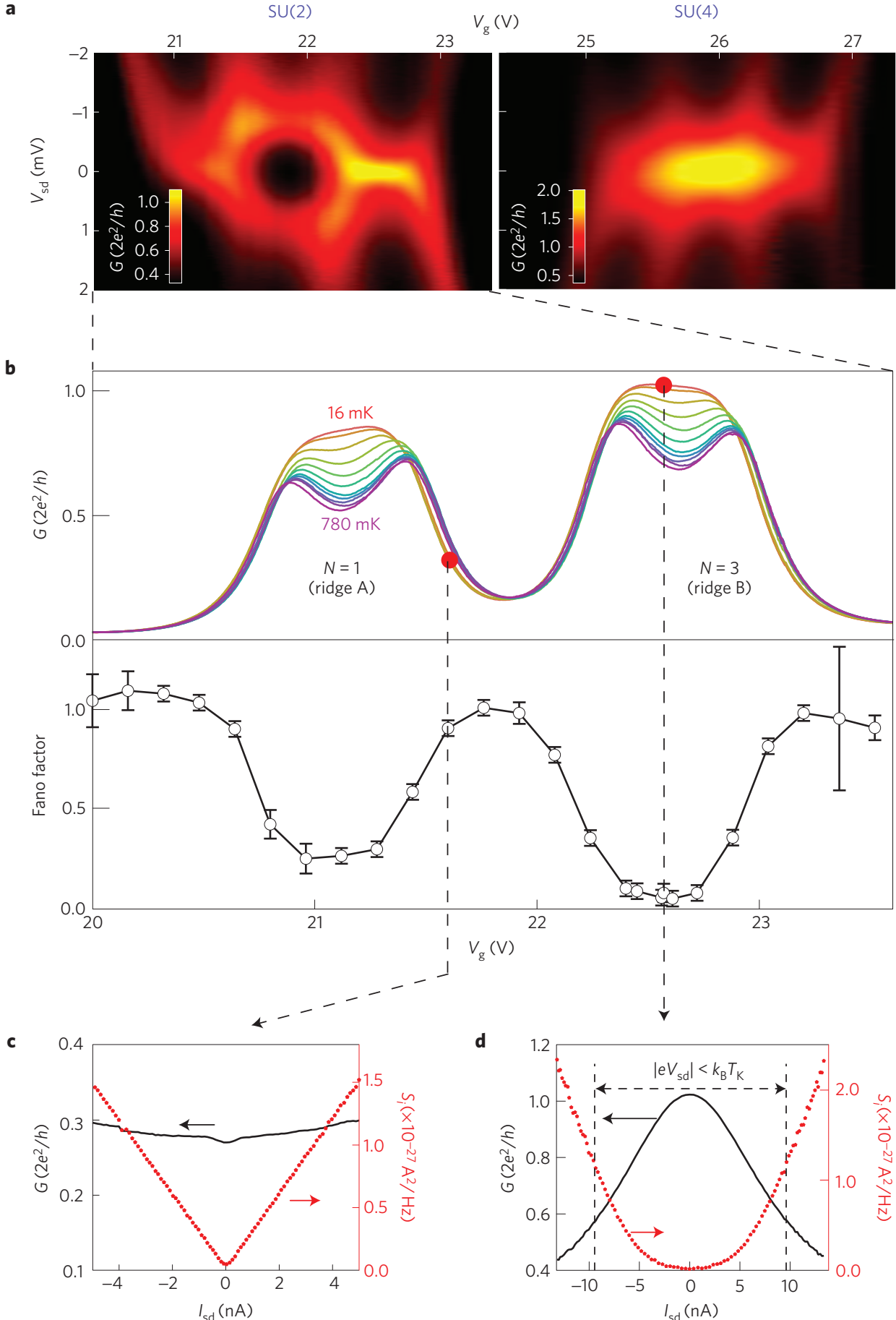

Figure 2 | Stability diagram and shot noise for the $S U(2)$ Kondo effect at $T=16 \mathrm{mK}$. $\mathbf{a}$, Two-dimensional plot of the conductance as a function of $V_{\text {sd }}$ and $V_{g}$. Kondo ridges appear as bright horizontal lines for $V_{s d}=0$. In the $S U(2)$ region two ridges show up for $N=1$ and $N=3$ electrons. For $S U(4)$ the Kondo ridge exists for $N=1,2$ and 3 electrons. Note that the conductance scale is $2 e^{2} / h$ for SU(2) whereas it is $2 \times 2 e^{2} / h$ for SU(4). An in-plane magnetic field of $0.08 \mathrm{~T}$ is applied to suppress superconductivity in the contacts. b. Upper panel: Cross-sections of the conductance of the SU(2) Kondo region at zero bias as a function of $V_{g}$ for different temperatures. Two Kondo ridges $A$ and $B$ are clearly visible. Lower panel: Fano factor extracted from the linear part of the current noise in the regime $e V_{s d} \ll k_{B} T_{K}\left(I_{s d} \leq 5 \mathrm{nA}\right)$ using the definition $S_{i}=2 e F\left|I_{s d}\right|$. Error bars take into account the uncertainty of the linear fit performed on slightly different current ranges in the limit $e V_{s d} \ll k_{B} T_{K} . c$. Conductance (black line) and noise (red dots) as a function of $I_{s d}$ for the blockaded region $(N=2)$. The noise is linear with $\left|I_{s d}\right|$, with a slope around 2 e. Outside the Kondo ridge, owing to the very small transmission, the quantum dot is close to the tunnelling regime characterized by a full Poissonian noise with $F=1$. d, Conductance (black line) and noise (red dots) as a function of $I_{\text {sd }}$ on the Kondo ridge $(N=3)$. The slope of $S_{i}$ around $I_{s d}=0$ is almost zero owing to the perfect transmission $(F=0.06)$.

are half transmitted $\left(T_{1}=T_{2}=0.5\right)$, yielding the same conductance $G_{\mathrm{Q}}$ as in the $\mathrm{SU}(2)$ symmetry. However, for $N=2$, current is transmitted through two perfect channels $\left(T_{1}=T_{2}=1\right)$, increasing the conductance to $G=2 G_{\mathrm{Q}}$. In this region the conductance hardly depends on temperature up to $800 \mathrm{mK}$, reflecting a large $T_{\mathrm{K}}$, as expected for the SU(4) symmetry ${ }^{24}$. This is confirmed by the full 
a
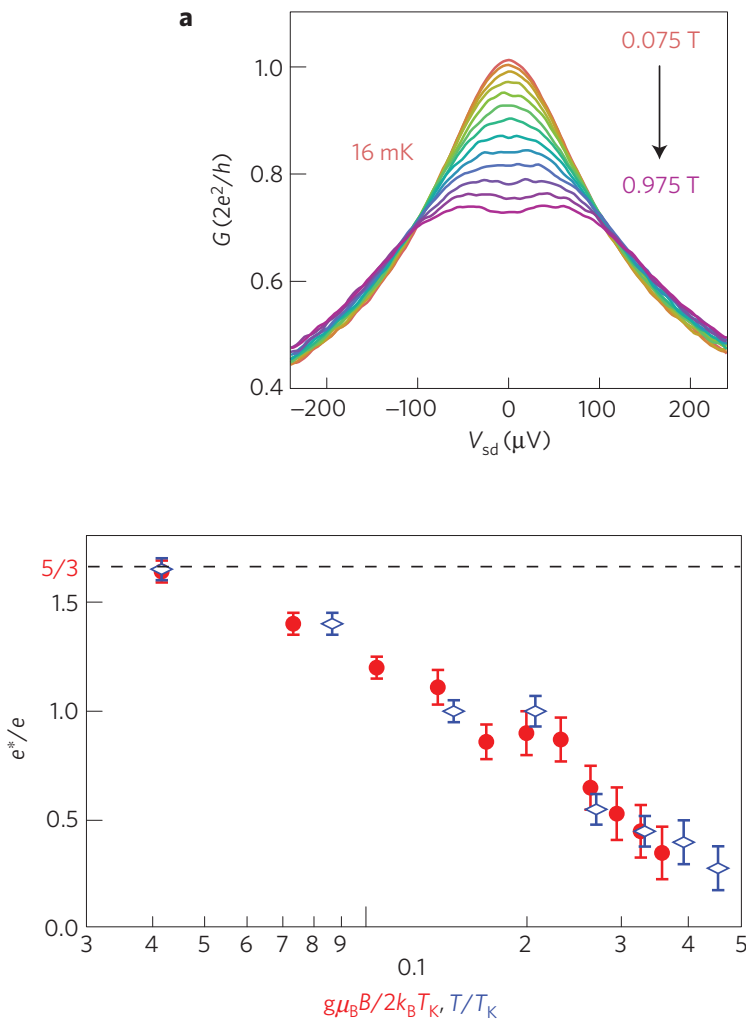

b
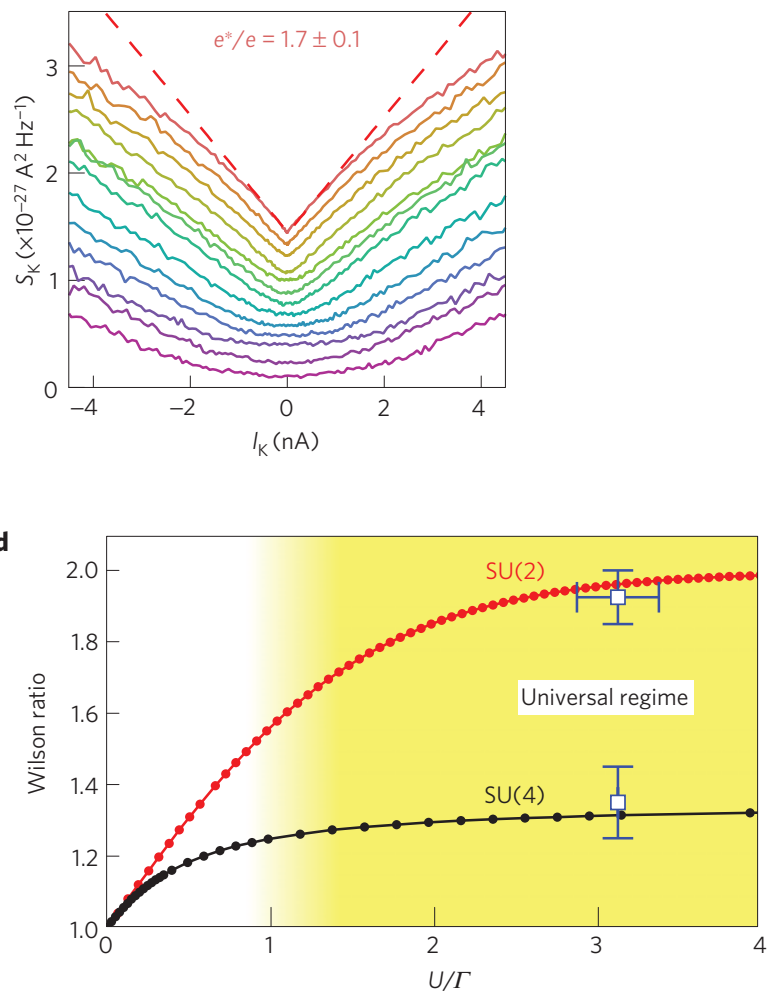

Figure 3 | Evolution of Kondo correlations with magnetic field and temperature. a, Evolution of the differential conductance from $0.075 \mathrm{~T}$ to $0.975 \mathrm{~T}$ in steps of $0.075 \mathrm{~T}$. $\mathbf{b}$, Kondo excess current noise $S_{K}$ as a function of backscattering current $I_{K}$ (see text for definition) for the same fields. The effective charge $e^{*}$ is extracted from the slope $S_{K} / 2 e l_{K}$ at low current. The dashed red line shows result of the linear fit, yielding $e^{*} / e=1.7 \pm 0.1$. The curves are shifted for clarity. $\mathbf{c}$, Effective charge as a function of $T / T_{K}$ and $g \mu_{B} B / 2 k_{B} T_{K}$ in a semi-log plot. Both can be described by the same line, which is a signature of a scaling law for $e^{*}(T, B)$ and suggests a logarithmic behaviour. Error bars take into account the uncertainty of the linear fit of $S_{K}$ as a function of $I_{K}$ performed on slightly different current ranges in the limit $e V_{\mathrm{sd}} \ll k_{\mathrm{B}} T_{\mathrm{K}}$. d, Wilson ratio as a function of $U / \Gamma$ calculated with the formula from ref. 15. For $\mathrm{SU}(2)$, vertical error bars represent the value for $R$ extracted from $e^{*}$ and from the scaling analysis of the conductance shown in the Supplementary Information. Horizontal error bar represents the value for $U / \Gamma$ extracted independently from the fit of Kondo temperature shown in the Supplementary Information. The crossing on the theoretical curve shows the agreement of our measurement with the extension of Fermi liquid theory out of equilibrium. For $\mathrm{SU}(4)$, the value of $R$ is extracted from $e^{*}$. The yellow part of the graph represents the region of universality: all the properties depend on a single parameter $T_{\mathrm{K}}$ for a given symmetry group ${ }^{11}$.

width of the curve $G\left(V_{\text {sd }}\right)$, which gives $T_{\mathrm{K}} \approx 11 \mathrm{~K}$ for $N=2$ and $T_{\mathrm{K}} \approx 17 \mathrm{~K}$ for $N=1$ or 3 .

The first result to emphasize is that the linear part of the current noise is qualitatively different from $\mathrm{SU}(2)$ and is a powerful experimental tool to distinguish the two symmetries ${ }^{14,18}$. The upper part of Fig. $4 \mathrm{~b}$ represents the conductance at $N=3$ for $\mathrm{SU}(2)$ and $\mathrm{SU}(4)$ as a function of the rescaled voltage $e V_{\text {sd }} / k_{\mathrm{B}} T_{\mathrm{K}}$. The two curves are barely distinguishable. However, the current noise shown on the lower part of Fig. $4 \mathrm{~b}$ is qualitatively different as it is almost zero for the $\mathrm{SU}(2)$ symmetry, whereas it is linear with $\left|I_{\text {sd }}\right|$ for $\mathrm{SU}(4)$. The linear noise is one order of magnitude stronger for SU(4) than for SU(2). Indeed, the Fano factor for two channels such that $T_{1}=T_{2}=0.5$ is $F=0.5$ for the $\mathrm{SU}(4)$ symmetry, whereas for $\mathrm{SU}(2)$ a single channel with $T_{1}=1$ yields $F=0$. The complete evolution of $F$ is summarized in the lower part of Fig. 4a. It changes from $F \approx 1$ outside the Kondo ridge to $F=0.5$ for $N=1$ and 3, reaching $F=0.07$ for $N=2$. This confirms that in the $N=2 \mathrm{SU}(4)$ case, transport takes place through two almost perfect channels $\left(T_{1}=T_{2}=1\right)$ without partition, yielding $F \approx 0$.

Finally we discuss $e^{*}$ and $R$ for the SU(4) symmetry at half filling $(N=2)$. $S_{\mathrm{K}}$ and $I_{\mathrm{K}}$ have been computed by using the same procedure as explained for $\mathrm{SU}(2)$. The result, shown in Fig. 4c, gives $e^{*} / e=1.45 \pm 0.1$ and $R=1.35 \pm 0.1$ from (equation (3)). The decreasing value for $R$ and $e^{*}$ reflects the increasing number of degenerated states. When degeneracy increases, electrons correlations become weaker and the non-interacting value is recovered for $\mathrm{SU}(n)$ when $n \rightarrow \infty$. The values for $e^{*}$ and $R$ are in good agreement with refs 14,15 (also see Fig. $3 d$ ), which predict $e^{*}=1.5$ and $R=4 / 3$, confirming that non-equilibrium Fermi liquid theory can be extended to more exotic classes of Fermi liquids.

Our experimental results emphasize three important points for the theory of Fermi liquids. First, in the linear regime they can be described as free quasi-particles, as the spirit of the theory teaches us. This allows one to clearly distinguish the different symmetry classes of Fermi liquids through shot noise measurements, whereas it is hardly possible from the conductance. Second, by probing the nonlinear noise, we have shown that out of equilibrium the residual interaction between quasi-particles shows up and creates a peculiar two-particle scattering, which bears the signature of the correlated nature of quantum liquids. Finally, the newly discovered out-ofequilibrium scaling law should trigger theoretical developments to deepen our understanding of universal behaviours in these liquids.

Received 26 March 2015; accepted 13 October 2015;

published online 23 November 2015

\section{References}

1. Mahan, G. D. Many-Particle Physics (Plenum Press, 1990).

2. Bloch, I., Dalibard, J. \& Nascimbène, S. Quantum simulations with ultracold quantum gases. Nature Phys. 8, 267-276 (2012). 
a
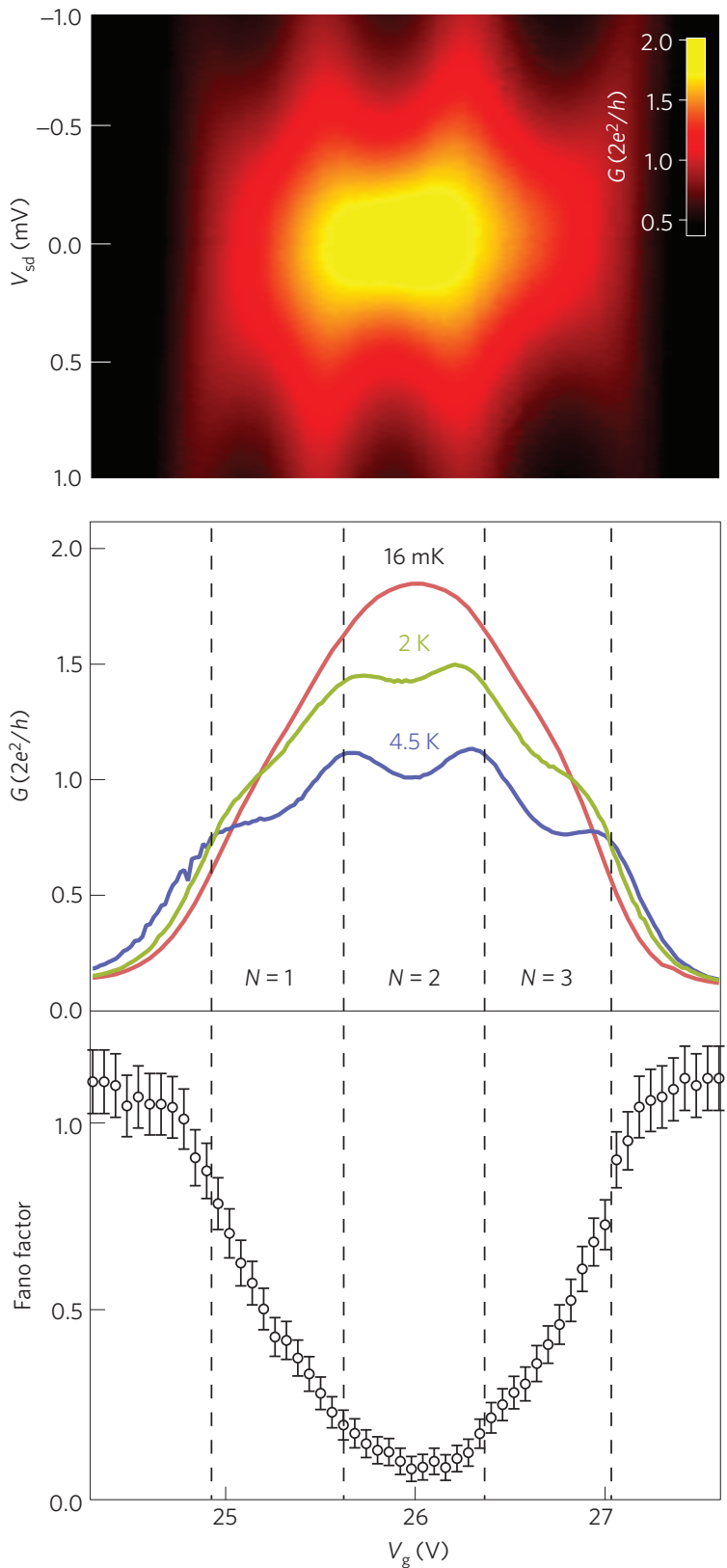

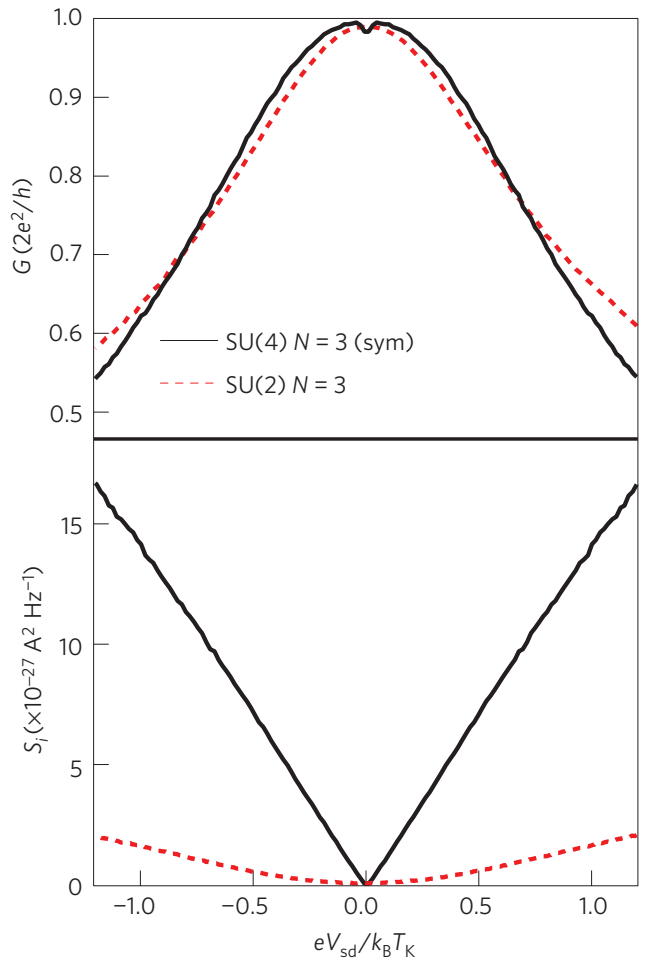

C

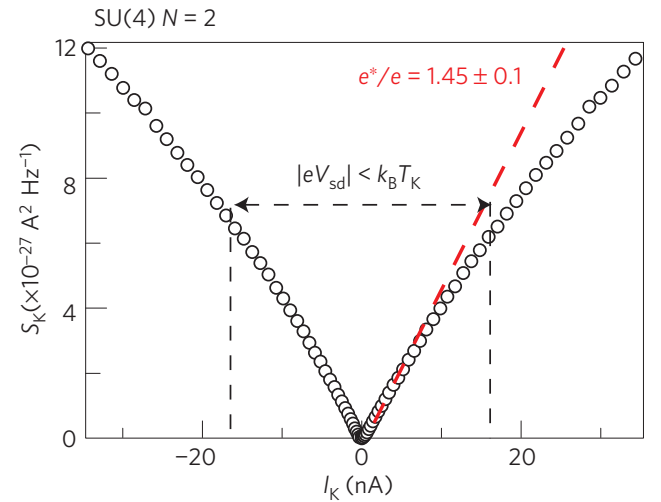

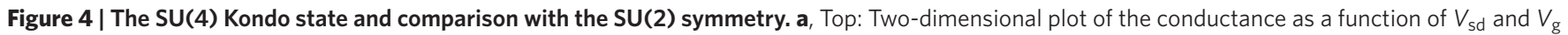
in the SU(4) state. Middle: cross-section of the conductance as a function of the gate voltage at $V_{\mathrm{sd}}=0$ measured at different temperatures. $G$ reaches $1.8 \times 2 e^{2} / h$ for the filling $N=2$. Bottom: Extracted Fano factor. Error bars come from the linear fit of $S_{\text {I }}$ as a function of $I_{\mathrm{sd}}$ in the limit eV $V_{\mathrm{sd}} \ll k_{\mathrm{B}} T_{\mathrm{K}}$. As expected it varies from $F \approx 0$ for $N=2$ to $F=0.5$ for $N=1$, and finally $F=1$ in the tunnelling regime (outside the Kondo ridge). $\mathbf{b}$, Comparison of the conductance (top) and the noise (bottom) for the SU(2) Kondo state and SU(4) at filling $N=3$. In both cases the conductance is the same and the two states are not distinguishable. At low voltage, the noise $S_{i}$ is qualitatively strongly different. For SU(2) the slope (Fano factor) is close to 0 , as the transmission is almost perfect $\left(T_{1}=1\right)$, whereas the linear part is very important in the SU(4) state, as transport occurs through two half transmitting channels $\left(T_{1}=T_{2}=0.5\right.$ ), yielding $F=0.5$. For clarity, as asymmetry with respect to $V_{\text {sd }}$ appears in the SU(4) state at large voltage, we compare only the symmetrized part of the conductance and the noise for $S U(4)$. We used the definition: $G^{\text {sym }}\left(V_{\mathrm{sd}}\right)=\left(G\left(V_{\mathrm{sd}}\right)+G\left(-V_{\mathrm{sd}}\right)\right) / 2$ and $S_{i}^{\text {sym }}\left(V_{\mathrm{sd}}\right)=\left(S_{i}\left(V_{\mathrm{sd}}\right)+S_{i}\left(-V_{\mathrm{sd}}\right)\right) / 2$. c, Kondo excess noise for the $S U(4)$ symmetry at filling $N=2$. A linear fit at low current using formula (2) gives $e^{*} / e=1.45 \pm 0.1$, yielding $R=1.35 \pm 0.1$

3. van der Wiel, W. G. et al. The Kondo effect in the unitary limit. Science 289, 2105-2108 (2000).

4. Jarillo-Herrero, P., Kong, J., Zant, H. S. J. V. D. \& Dekker, C. Orbital Kondo effect in carbon nanotubes. Nature 434, 484-488 (2005).

5. Makarovski, A., Zhukov, A., Liu, J. \& Finkelstein, G. SU(2) and SU(4) Kondo effects in carbon nanotube quantum dots. Phys. Rev. B 75, 241407 (2007)

6. Cleuziou, J. P., NGuyen, N. G., Florens, S. \& Wernsdorfer, W. Interplay of the Kondo effect and strong spin-orbit coupling in multihole ultraclean carbon nanotubes. Phys. Rev. Lett. 111, 136803 (2013).
7. Schmid, D. R. et al. Broken SU(4) symmetry in a Kondo-correlated carbon nanotube. Phys. Rev. B 91, 155435 (2015).

8. Delattre, T. et al. Noisy Kondo impurities. Nature Phys. 5, 208-212 (2009).

9. Nozières, P. A "Fermi-Liquid" description of the Kondo problem at low temperatures. J. Low Temp. Phys. 17, 31-42 (1974).

10. Sela, E., Oreg, Y., von Oppen, F. \& Koch, J. Fractional shot noise in the Kondo regime. Phys. Rev. Lett. 97, 086601 (2006).

11. Oguri, A. Fermi liquid theory for the nonequilibrium Kondo effect at low bias voltages. J. Phys. Soc. Jpn 74, 110-117 (2005). 
12. Gogolin, A. \& Komnik, A. Full counting statistics for the Kondo dot in the unitary limit. Phys. Rev. Lett. 97, 016602 (2006).

13. Mora, C., Leyronas, X. \& Regnault, N. Current noise through a Kondo quantum dot in a SU(N) Fermi liquid state. Phys. Rev. Lett. 100, 036604 (2008).

14. Mora, C., Vitushinsky, P., Leyronas, X., Clerk, A. \& Le Hur, K. Theory of nonequilibrium transport in the SU(N) Kondo regime. Phys. Rev. B 80, 155322 (2009).

15. Sakano, R., Fujii, T. \& Oguri, A. Kondo crossover in shot noise of a single quantum dot with orbital degeneracy. Phys. Rev. B 83, 075440 (2011).

16. Zarchin, O., Zaffalon, M., Heiblum, M., Mahalu, D. \& Umansky, V. Two-electron bunching in transport through a quantum dot induced by Kondo correlations. Phys. Rev. B 77, 241303 (2008).

17. Yamauchi, Y. et al. Evolution of the Kondo effect in a quantum dot probed by shot noise. Phys. Rev. Lett. 106, 176601 (2011).

18. Egger, R. What the noise is all about. Nature Phys. 5, 175-176 (2009).

19. Kondo, J. Resistance minimum in dilute magnetic alloys. Prog. Theor. Phys. 32, 37-49 (1964)

20. Blanter, Y. M. \& Büttiker, M. Shot noise in mesoscopic conductors. Phys. Rep. 336, 1-166 (2000).

21. Kasumov, Y. et al. CVD growth of carbon nanotubes at very low pressure of acetylene. Appl. Phys. A 88, 687-691 (2007).

22. Arakawa, T., Nishihara, Y., Maeda, M., Norimoto, S. \& Kobayashi, K. Cryogenic amplifier for shot noise measurement at $20 \mathrm{mK}$. Appl. Phys. Lett. 103, 172104 (2013).

23. Sakano, R., Oguri, A., Kato, T. \& Tarucha, S. Full counting statistics for SU(N) impurity Anderson model. Phys. Rev. B 83, 241301 (2011).
24. Galpin, M. R., Logan, D. E. \& Krishnamurthy, H. R. Quantum phase transition in capacitively coupled double quantum dots. Phys. Rev. Lett. 94, 186406 (2005).

\section{Acknowledgements}

We appreciate discussions with $\mathrm{H}$. Bouchiat and R. Yoshii. This work was partially supported by a Grant-in-Aid for Scientific Research (S) (No. 26220711), JSPS KAKENHI (No. 26400319, 25800174 and 15K17680), Invitation Fellowships for Research in Japan from JSPS, Grant-in-Aid for Scientific Research on Innovative Areas 'Fluctuation \& Structure' (No. 25103003) and 'Topological Materials Science' (KAKENHI Grant No. 15H05854), the Program for Promoting the Enhancement of Research Universities from MEXT, and Yazaki Memorial Foundation for Science and Technology, and the French programmes ANR DYMESYS (ANR2011-IS04-001-01) and ANR MASH (ANR-12-BS04-0016). K.K. acknowledges the stimulating discussions in the meeting of the Cooperative Research Project of RIEC, Tohoku University.

\section{Author contributions}

All the authors contributed to the analysis of the data and commented on the manuscript. M.F., R. Delagrange., R.W. and R. Deblock designed the sample. M.F., T.A., T.H. and R.F. performed the noise experiments and analysed the data. M.F. and K.K. planned and supervised the research.

\section{Additional information}

Supplementary information is available in the online version of the paper. Reprints and permissions information is available online at www.nature.com/reprints.

Correspondence and requests for materials should be addressed to M.F. or K.K.

\section{Competing financial interests}

The authors declare no competing financial interests. 